\title{
Improvements to Response-Surface Based Vehicle Design Using a Feature-Centric Approach
}

\author{
David Thompson ${ }^{1}$, Srinivasan Parthasarathy ${ }^{2}$, Raghu Machiraju ${ }^{2}$, \\ and Scott Lawrence ${ }^{3}$ \\ ${ }^{1}$ Department of Aerospace Engineering and Computational Simulation and Design Center, \\ Mississippi State University, MS \\ dst@erc.msstate.edu \\ ${ }^{2}$ Computer and Information Science, The Ohio State University, Columbus, $\mathrm{OH}$ \\ \{raghu,srini\}@cis.ohio-state.edu \\ ${ }^{3}$ Systems Analysis Branch, Ames Research Center, NASA, Moffett Field, CA \\ scott.L.Lawrence@nasa.gov
}

\begin{abstract}
In this paper, we present our vision for a framework to facilitate computationally-based aerospace vehicle design by improving the quality of the response surfaces that can be developed for a given cost. The response surfaces are developed using computational fluid dynamics (CFD) techniques of varying fidelity. We propose to improve the quality of a given response surface by exploiting the relationships between the response surface and the flow features that evolve in response to changes in the design parameters. The underlying technology, generalized feature mining, is employed to locate and characterize features as well as provide explanations for feature-feature and feature-vehicle interactions. We briefly describe the components of our framework and outline two different strategies to improve the quality of a response surface. We also highlight ongoing efforts.
\end{abstract}

\section{Introduction}

Design studies for advanced aerospace vehicles are typically performed using a suite of computational fluid dynamics (CFD) simulation tools. An integral component of these design studies is the development of response surfaces describing vehicle performance characteristics as functions of the various geometrical design parameters. Essentially, the response surfaces provide a method for abstracting the results of a simulation [1]. A response surface may be generated using tools of differing fidelity, i.e., variable complexity modeling [1]. Because of various factors associated with the simulations, e.g., incomplete convergence of iterative methods, round-off errors, and truncation errors [2], numerical noise may be present in the response surfaces. When such noise occurs, the optimization procedure may be slow to converge or converge to an erroneous local extrema in the design space. This can be partially obviated through the use of response surface modeling [1]. The response surfaces typically include force and moment components and consist of the outputs from a series of CFD simulations. Continuous approximations to the response surfaces are obtained through a surface fitting process. These continuous approximations are then employed 
in a design algorithm to determine a geometrical parameter set that results in a set of vehicle characteristics that satisfy specified criteria.

Response surfaces can also be employed to optimize vehicle trajectories. In this context, the design parameters include vehicle attitude, control surface deflections, etc. Knowledge about the presence and location of flow features would provide guidance on judicious use of the available analysis tools as well as provide an understanding of the level of confidence that can be placed on the computational results in various regions of the parameter space.

Unfortunately, the quality of a given response surface is difficult to ascertain. This is primarily due to the fact that development of analysis techniques for CFD data has not kept pace with our ability to generate massive data sets. To address the difficulties associated with analyzing evolutionary data generated by large-scale simulations of complex phenomena, we are developing a framework that we term generalized feature mining. In the context of this effort, i.e., aerospace vehicle design, we are interested in how flow features, which influence the performance of the vehicle, evolve in response to changes in the design parameters. Hence, the features, which may be static features in the sense that they do not evolve over time, do evolve as the design parameters are modified.

\subsection{Motivation}

We can formalize the notion of response surfaces by letting $\mathbf{a}=\left\{a_{1}, \ldots, a_{n}\right\}$ be the set of $n$ parameters that are considered in the design of a vehicle. These typically include the geometrical characteristics of the configuration of interest. Let $\mathbf{f}(\mathbf{a})=\left\{\psi_{1}(\mathbf{a}), \ldots, \psi_{\mathrm{m}}(\mathbf{a})\right\}$ be $m$ quantities of interest such as force and moment components. Each of the $\psi_{l}(\mathbf{a})$ form a response surface. Vehicle design attempts to find a set of design parameters a which yields a desirable vector $\mathbf{f}(\mathbf{a})$ for certain operational conditions. These response surfaces are obtained by computing the flow field and the desired quantities $\psi_{\mathrm{r}}$ for a given configuration a. It should be noted that some response surfaces, such as configuration gross weight, depend only on the geometry and material properties and are independent of the flow simulation.

What is lacking from the approaches described above is a true "cause and effect" perspective for vehicle design. Changes in the response surfaces $\psi_{i}(\mathbf{a})$ are correlated only to changes in the design parameter vector a. In our approach, we seek explanations for the behavior of a response surface $\psi_{\mathrm{i}}$. Desirable conditions may occur in due to the presence (or absence) of specific flow phenomena. Understanding the properties of these phenomena and their interactions with the vehicle or other flow features provides more insight than correlations with the design parameters. We believe that these insights will lead to more cost effective methods for estimating the response surface, thereby reducing design cost.

\subsection{Outline of Paper}

In Section 2 we describe the basic components of our feature-centric design framework. Section 3 describes two scenarios which can exploit generalized feature mining in a design context. In Section 4, we describe related work and in Section 5 
we describe on-going work in to develop generalized feature mining technologies. Finally, we include a summary of the paper in Section 6.

\section{Components of Generalized Feature Mining}

The generalized feature mining framework will accept as input a set of CFD simulation results $\mathbf{S}$ and the corresponding set of designs $\mathbf{A}$ that produced these results. Additional inputs include a definition of the material properties and a specification of flight and environmental conditions. There are four components that must be developed to accomplish our stated objective:

- Extraction of the feature set: The simulation results $\mathbf{S}$ will be processed to extract features F. Shape descriptors will be used to characterize the features. Here, a shape descriptor for a given feature is an abstract representation of the feature including its geometrical and dynamical characteristics.

- Explanation of feature-feature and feature-vehicle interactions: In addition to shape descriptors, generalized feature mining will generate explanations $\mathbf{E}$ correlated with changes in the design a through the evolution of the feature set $\mathbf{F}$.

- Characterization of a response surface: Once a response surface $\mathbf{R}$ is generated, it will be characterized qualitatively and quantitatively using shape descriptors $\mathbf{F}$ and explanations $\mathbf{E}$.

- Estimation of the response surface: New points on the response surface will be estimated based on the response surface characterization. This will facilitate determination of whether an additional computation needs to be performed to locally 1) refine the response surface or 2) improve its accuracy user a higher fidelity method.

\section{Application to Aerospace Vehicle Design}

We envision that our framework may be employed for aerospace vehicle design via two different design-of-experiment paradigms. Both approaches are designed to improve the quality of a response surface that can be generated for a given cost resulting in a higher confidence design. We describe them below:

- The quality of a response surface may be improved locally through the use of higher fidelity methods. An initial response surface is developed using lower fidelity (less expensive) computational tools. Our framework is then employed to identify regions of the response surface whose associated feature catalogs, i.e., flow features, feature-feature interactions, and featurevehicle interactions, suggest that a higher fidelity simulation tool should be employed. The cost of generating the response surface is decreased since the number of expensive simulations needed to adequately define the response surface is reduced. 
- The quality of a response surface may be improved locally through refinement. An initial response surface is developed using a relatively coarse sampling. Our framework is then employed to identify regions of the response surface whose associated feature catalogs suggest are undersampled. Additional simulations of appropriate fidelity may be performed at the identified design points. The cost of generating the response surface is again decreased since the number of expensive simulations needed to adequately define the response surface is reduced.

We also suggest that it may be possible to employ the two different paradigms in tandem to adaptively generate a high quality response surface for a reduced cost.

\section{Related Work}

In this section we report on work related to the current effort. A timely book on scientific and engineering data mining techniques edited by Grossman, Kamath, Kegelmeyer, Kumar, and Namburu [3] discusses many issues relevant to this effort. Kamath provides a beautiful overview of the area along with some of the inherent challenges (Chapter 1). Marusic and his associates (Chapter 13) and Han and his associates (Chapter 14) consider the problem of mining computational fluid dynamics simulations using standard data mining techniques in conjunction with basic physical modeling of entities or features. The work by the authors Machiraju, Thompson and colleagues (Chapter 15) on the EVITA project is a precursor to the current effort. Han and colleagues (Chapter 25), Sekhar and colleagues (Chapter 26), and Lesage and Pace (Chapter 24) report on the usefulness of spatial data analysis and survey such techniques in the context of engineering and scientific applications. Additionally techniques for mining astronomical data (Chapters 5 and 6) and earth science observations (Chapter 10) are also reported in this book. However, few of the above methods actually account for the structural or physical properties of the data. Also, none of them address the application of these techniques to design.

Yip and Zhao [4] proposed a framework called Spatial Aggregation (SA). They relied on segmentation (or spatial aggregation) to cluster both physical and abstract entities and constructed imagistic solvers to gain insights about physical phenomena. Our paradigms for feature mining have close relationship to the SA framework. The work by Ester and colleagues [5] on trend detection in spatial databases and spatial database primitives are also quite relevant to our work on spatial associations. The work by Graves and colleagues [6] is also relevant but focused mainly on feature extraction and mining of image data (satellite). It should be noted that the ADAM framework is extensible and it may be possible to incorporate many of the techniques described here.

\section{Ongoing Work}

In this section we describe our preliminary efforts in data mining and effort towards realizing a framework for generalized feature mining. Our combined preliminary 


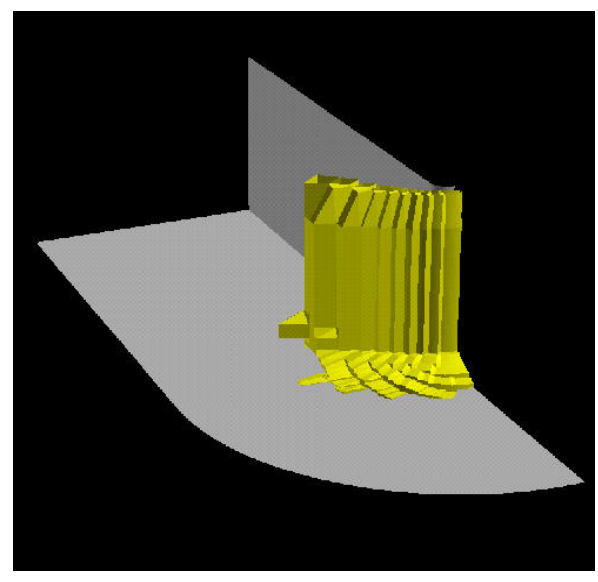

(a)

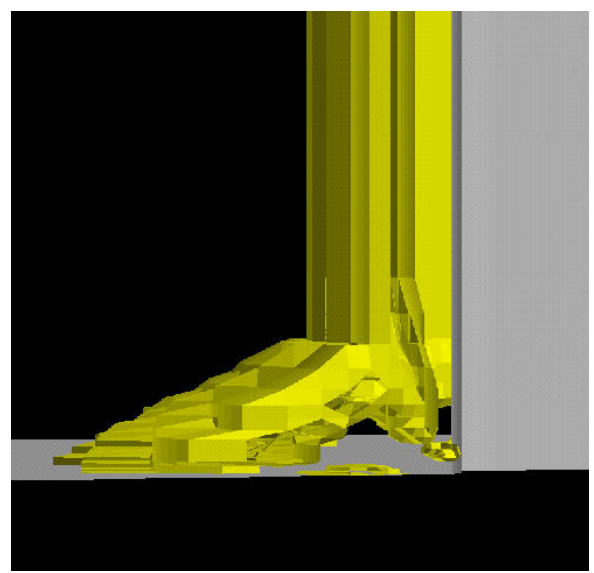

(b)

Fig. 1. Point Classification Techniques (a) The Blunt Fin Example (b) A zoomed version

work on feature mining has been reported in the literature [7-9]. It should be noted that our work spans two application domains, namely CFD and molecular dynamics. Because of the paucity of space and vehicle design context, we focus on work applicable to CFD simulation data.

We first describe two distinct feature mining paradigms. The common thread is that both are bottom-up feature constructions based on underlying physically based criteria. They consist essentially of similar steps; however, the order of the steps is different. The first paradigm, based on point classification, is suitable for features that can be defined and detected with local operators such as shocks and defects. The second paradigm, based on an aggregate classification, is suitable for features with a more global influence such as vortices. More details can be found in [8-9].

As an example of the point classification paradigm, we consider shock wave detection in flow fields. A shock is an abrupt compression wave that may occur in fluid flows when the velocity of the fluid exceeds the local speed of sound. The properties of shocks are can be exploited to develop highly discriminating shock detection algorithms [10]. Figure 1(a) shows a detached oblique shock that wraps around the blunt fin in the standard blunt fin/flat plate flow field solution. Figure 1(b) shows the symmetry plane which intersects a lambda-shock.

We recently developed vortex detection and verification algorithms that exploit the aggregate classification paradigm [11,12]. For the local detection operator, a combinatorial labeling scheme is employed. Our detection algorithm labels the velocity vectors at the grid points and identifies grid cells that are likely to contain the vortex core. Our technique then segments candidate vortex core regions by aggregating points identified from the detection step. We then classify (or verify) these candidate core regions based on the existence of swirling streamlines surrounding them. In Figure 2 we show the vortex cores and a detailed view of the vortical flow near the fin/plate intersection in the left and center images, respectively. The aggregate classification paradigm identifies individual points as being probable candidate points in a feature and then aggregates them. The verification algorithm 


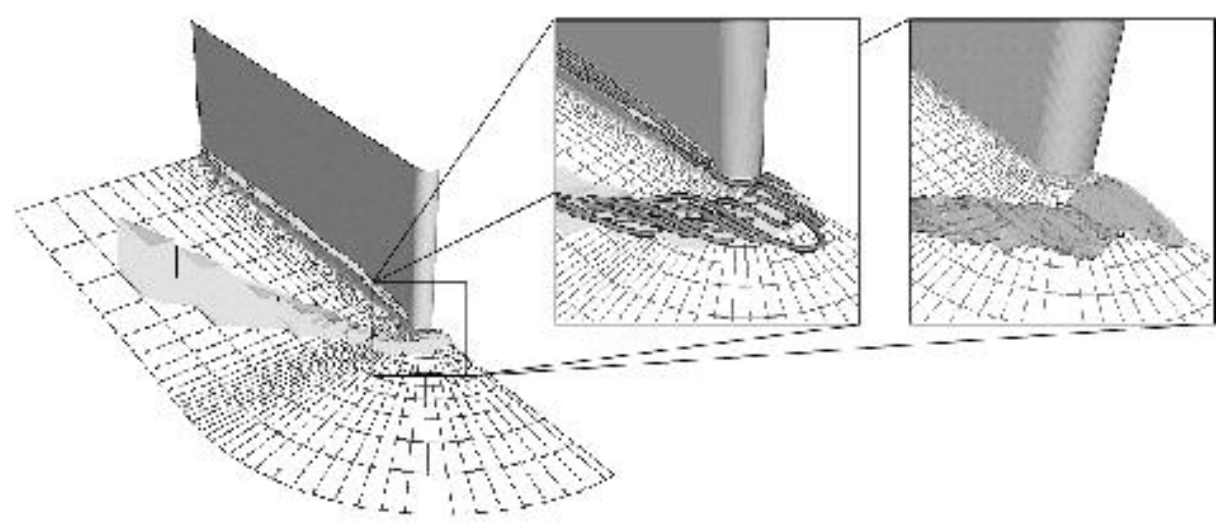

Fig. 2. Aggregate Classification Techniques. We use the Blunt Fin example again. The first inset shows the verification algorithm at work. The second inset shows the modeling of the swirling region as a collection of frusta.

then is applied to the aggregate using physics-based non-local criteria to determine whether the aggregate is actually a feature. In this case, the binary feature classification is applied to an aggregate of points. Features that are regional in nature, such as a vortex, benefit from the aggregate classification approach.

Having extracted the features, we now describe the next step: characterizing them using geometric shape attributes. The swirling region of a vortex can be characterized using a sequence of elliptical frusta (a conical frusta with two ends as ellipses) [8]. Starting from the upstream extent of the vortex core, the modeling process seeds a set of swirling streamlines surrounding the core region. Each elliptical frustum is oriented along the longest segment of the vortex core that does not curve by more than a userspecified amount. We illustrate the adaptive and hierarchical shape characterization process in the right image of Figure 2. The elliptical shape of the primary vortex as well as its curved core provide visual validation of the choice of the geometric descriptors.

We now describe an ongoing data mining effort that has, to date, been employed for the discovery of substructures in large molecules. Motifminer, is a novel scalable toolkit to efficiently discover frequently occuring spatial and structural relationships in scientific data [13]. The technique relies on range pruning (limits the search for viable spatial relationships) and candidate pruning (similar to candidate pruning in association rule mining but with a spatial twist) for pruning the search space of possible frequent structures. We also rely on fuzzy recursive hashing, a technique akin to geometric hashing, for rapid matching of frequent spatial relationships (to determine frequency of occurrence).

In addition feature characterization, much work is being conducted towards tracking of these swirling regions. Also, work has begun toward determining spatial associations and using the gained knowledge to glean explanations about the underlying phenomena. 


\section{Summary}

We have described our vision for a feature-centric framework to improve simulationbased aerospace vehicle design. We proposed two different strategies, both based on generalized feature mining, to improve the quality of response surfaces employed in the design process by exploiting the relationships between the response surface and the flow features that evolve in response to changes in the design parameters. Also included were brief discussions of the various components of our framework and current efforts to realize these components. We believe our approach may prove to be a viable cost effective alternative to current automated design techniques.

\section{References}

1. Giunta, A., Golividov, O., Knill, D. L., Grossman, B., Haftka, B., Mason, W., Watson, L.: Multidisciplinary Design Optimization of Advanced Aircraft Configurations. 15th International Conference on Numerical Methods in Fluid Dynamics, Monterey, CA, June 1996.

2. Baker, C., Grossman, B., Haftka, R., Mason, W., Watson, T.: High-Speed Civil Transport Design Space Exploration Using Aerodynamic Response Surface Approximations, J. Aircraft, 39(2):215-220, 2002.

3. Grossman, R., Kamath, C., Kegelmeyer, P., Kumar, V., Namburu, R.: Data Mining for Scientific and Engineering Applications. Kluwer Academic Press, 2001.

4. K. Yip and F. Zhao: Spatial Aggregation: Theory and Applications. J. of Artificial Intelligence Research, 5:1-26, Aug 1996.

5. Ester, R., Frommelt, A., Kriegel, H., Sander, A.: Algorithms for characterization and trend detection in spatial databases. KDD, 1998.

6. Ramachandran, R., Conover, H., Graves, S., Keiser, K.: Algorithm development and mining (ADAM) system for earth science applications. Second Conference on Artificial Intelligence, $80^{\text {th }}$ American Meteorogical Society Annual Meeting, 2000.

7. Machiraju, R., Parthasarathy, S., Thompson, D. S., Wikins, J., Gatlin, B., Choy, T. S., Richie, D., Jiang, M., Mehta, S., Coatney, M., Barr, S., Hazzard, K.: Mining Complex Evolutionary Phenomena. In H. Kargupta, editor, Data Mining for Scientific and Engineering Applications. MIT Press, 2003.

8. Jiang, M., Choy, T. S., Mehta, S., Coatney, M., Barr, S., Hazzard, K., Richie, D., Parthasarathy, S., Machiraju, R., Thompson, D., Wilkins, J., Gatlin, B.: Feature Mining Algorithms for Scientific Data. In Proceedings of SIAM Data Mining Conference, pages 13-24,May 2003.

9. Thompson, D., Machiraju, R., Jiang, M., Nair, J., Craciun, G., Venkata, S.: Physics-Based Feature Mining for Large Data Exploration. In IEEE Computing in Science and Engineering, 4(4):22-30, July-August 2002.

10. Lovely, D., Haimes, R.: Shock Detection from Computational Fluid Dynamics Results. AIAA Paper 99-3285, AIAA 14th Computational Fluid Dynamics Conference, June 1999.

11. Jiang, M., Machiraju, R., Thompson, D.: Novel Approach to Vortex Core Region Detection, In Proceedings of Joint Eurographics-IEEE TCVG Symposium on Visualization, pages 217-225, May 2002.

12. Jiang, M., Machiraju, R., Thompson, D.: Geometric Verification of Swirling Features in Flow Fields. In Proceedings of IEEE Visualization '02, pages 307-314, October 2002.

13. Parthasarathy, P., Coatney, M.: Efficient discovery of common substructures in acromolecules. In Proceedings of IEEE International Conference on Data Mining, 2002. 